\title{
Simulación clínica de alto realismo: una experiencia en el pregrado
}

\author{
Javier Riancho, José M. Maestre, Ignacio del Moral, José A. Riancho
}

Introducción. La simulación con modelos de alto realismo se utiliza a menudo en la formación de los profesionales sanitarios. Sin embargo, son escasas las experiencias en el pregrado. El objetivo de este trabajo fue conocer la factibilidad y la aceptación de su aplicación con estudiantes de sexto curso de la licenciatura de Medicina.

Materiales y métodos. Se diseñaron ocho escenarios que simulaban problemas clínicos frecuentes para su desarrollo con maniquíes de alto realismo. Los estudiantes se dividieron en grupos de 6-8 sujetos, cada uno de los cuales atendió dos casos durante 30 minutos. Posteriormente se llevó a cabo un análisis reflexivo durante 25-40 minutos. La actividad se repitió en dos años consecutivos. Al final se recabó la opinión de los estudiantes mediante encuestas anónimas.

Resultados. La actividad fue valorada muy positivamente por los estudiantes, quienes la consideraron como 'útil' (4,8 y 4,9 puntos sobre 5) e 'interesante' (4,9 y 4,9 puntos). El tiempo preciso para preparar cada escenario fue de unas 3 horas. Fueron necesarias una jornada completa de un profesor, un técnico y un enfermero para que un colectivo de unos 40 estudiantes se expusiera a dos casos clínicos.

Conclusiones. Esta experiencia piloto sugiere que la simulación de alto realismo es factible en el pregrado, supone un consumo razonable de recursos y tiene una elevada aceptación por parte de los estudiantes. No obstante, se necesitan otros estudios que confirmen la impresión subjetiva de que resulta útil para potenciar el aprendizaje de los alumnos y su competencia clínica.

Palabras clave. Clínica médica. Medicina clínica. Pregrado. Simulación.

\section{Realistic clinical simulation: an experience with undergraduate medical students}

Introduction. Realistic clinical simulation is commonly used with physicians and other health professionals. However it has been rarely used with undergraduate students. The aim of this study was to explore its feasibility and acceptance with medical students.

Materials and methods. Eight clinical scenarios representing common acute problems in medical practice were designed and implemented using a realistic manikin. In two consecutive years, groups of 6-8 students attended two cases during 30 min, followed by a 25-40 period for assessment and feedback. Students opinion was obtained by anonymous questionnaires.

Results. Students considered the activity both 'useful' ( 4.8 and 4.9 points in a 5-point scale) and 'interesting' ( 4.9 and 4.9 points). Designing each scenario required about 3 hours. During a work day of a teacher, a nurse and a technician, 40 students were exposed to two scenarios.

Conclusions. This pilot study suggest that realistic clinical simulations is both feasible and well-accepted by medical students, with reasonable resource requirements. Nevertheless, other studies are needed to confirm their usefulness to improve student learning and clinical performance.

Key words. Clinical medicine. Medical students. Simulation. Undergraduate.

\section{Introducción}

Tradicionalmente, el aprendizaje de la medicina, como el de otras profesiones dedicadas a aplicar unos conocimientos previos a la resolución de situaciones con elevada variabilidad, se ha basado en la observación y posterior actuación del alumno bajo la tutela del maestro. Así, en la concepción osleriana, el aprendizaje de la medicina tiene lugar esencialmente en la cabecera de la cama, a través del contacto directo con el paciente. Sin cuestionar la indudable eficacia de este método, algunas características de las organizaciones sanitarias actuales comprometen su aplicación y efectividad. Entre ellas,
Servicio de Neurología (J. Riancho); Servicio de Medicina Interna (J.A. Riancho); Hospital Universitario Marqués de Valdecilla. Hospital virtual Valdecilla (J.M. Maestre, I. del Moral). Departamento de Medicina y Psiquiatría; Universidad de Cantabria (J.A. Riancho). Santander, Cantabria, España.

Correspondencia: Dr. José A. Riancho. Departamento de Medicina y Psiquiatría. Universidad de Cantabria. Avda. Valdecilla, s/n. E-39008 Santander (Cantabria). Fax: +34942201665

E-mail: rianchoj@unican.es

Agradecimientos: Alejandro Martínez, Saúl García, Verónica Hoz y Elena Rojo (Hospital virtual Valdecilla).

Conflicto de intereses: No declarado.

Conflict of interests: None declared.

Cㄷ 2012 Educación Médica 
el manejo de pacientes cada vez más graves y la realización de complejas técnicas de diagnóstico y tratamiento, donde un equipo multiprofesional desarrolla varias tareas simultáneas, en un entorno dinámico e incierto donde la docencia difícilmente encuentra un lugar. Además, la elevada carga asistencial y la presión por acortar las estancias de los pacientes en los hospitales limitan el tiempo disponible para cada paciente y para la enseñanza de la medicina. Por otra parte, existe una mayor concienciación de los riesgos legales de la asistencia y ha aumentado la preocupación social por la seguridad de los pacientes cuando los estudiantes toman decisiones clínicas o realizan procedimientos sobre ellos [1,2]. Otro factor a considerar es que los encuentros clínicos están determinados por las necesidades del paciente; no se ajustan necesariamente al programa docente o los objetivos de aprendizaje. Desde luego, todo ello colisiona con los criterios de construcción del Espacio Europeo de Educación Superior, entre los cuales las actividades prácticas desempeñan un papel preeminente.

En este entorno ha surgido la simulación clínica como un método de aprendizaje complementario al resto de estrategias docentes que da respuesta a muchas de las necesidades planteadas. La simulación permite practicar en un entorno realista sin riesgo para pacientes y profesionales, facilita la estandarización de los contenidos docentes, contribuye a detectar deficiencias formativas y promueve la integración de conocimientos y habilidades clínicas complejas. Todo ello en un ambiente docente adecuado para los adultos, basado en el aprendizaje a partir de la propia experiencia y la reflexión personal [3].

La simulación clínica puede llevarse a cabo con diversos sistemas, en función de los objetivos perseguidos, incluyendo programas informáticos, simuladores de tareas para el aprendizaje de técnicas diagnósticas o terapéuticas, maniquíes de diversas características o pacientes simulados por actores. Un tipo particular de simulación es la basada en los maniquíes de alta fidelidad, que reproducen muchas de las características de los pacientes y posibilitan recrear escenarios de elevado realismo. La simulación clínica se utiliza con éxito en actividades de especialización y formación continuada, y en particular en el entrenamiento para el manejo de situaciones críticas en el ámbito hospitalario [4]. Sin embargo, apenas existen experiencias de su aplicación en el pregrado [5]. Por ello, nos ha parecido interesante describir una experiencia piloto de aplicación de estos sistemas en la docencia de la clínica médica a estudiantes de la licenciatura de Medicina, con el objetivo de conocer su factibilidad y aceptación.

\section{Materiales y métodos}

\section{Docencia de clínica médica}

En nuestra facultad, la clínica médica se imparte en el último año de la licenciatura. Su objetivo general es que los estudiantes recapitulen e integren los conocimientos adquiridos en cursos anteriores, en particular los relativos a las materias de semiología, fisiopatología y patología médica, y apliquen activamente esos conocimientos en un entorno de práctica real, de manera que sean capaces de abordar los problemas más frecuentes de la práctica médica. La docencia de la asignatura se articula a través de la discusión en el aula de casos de pacientes reales con problemas comunes del ámbito de la patología médica (fiebre, dolor torácico, disnea, dolor abdominal, hiperglucemia...) y de la rotación, durante dos periodos de siete semanas, por dos servicios del área de medicina del hospital o en un centro de salud. Los estudiantes reciben además algunos seminarios específicos (p. ej., sobre auscultación cardiaca) y pueden utilizar los modelos y maquetas del taller de habilidades clínicas. La evaluación se lleva a cabo combinando la valoración otorgada por el tutor durante las rotaciones, la exposición de un caso clínico y una prueba final que combina un examen escrito acerca del abordaje de casos clínicos de complejidad moderada y un examen objetivo estructurado con ocho estaciones.

\section{Hospital virtual Valdecilla (HvV)}

La misión general del $\mathrm{HvV}$ es mejorar la seguridad del paciente y hacer más eficaz el cuidado de la salud mediante el entrenamiento de los profesionales, la realización de investigaciones innovadoras y la promoción del trabajo en equipos clínicos de alto rendimiento, todo ello a través de la simulación clínica en todas sus aplicaciones. En las instalaciones se reproducen desde una habitación de una planta de hospital o de un servicio de urgencias, hasta un consultorio de atención primaria o un quirófano, y los materiales habitualmente disponibles en ellos (monitores, sistemas de venoclisis, etc.). Para sustituir a los pacientes se utilizan, dependiendo de los objetivos docentes, maniquíes de alta fidelidad controlados por ordenador (METI ECS ${ }^{\circledR}$ y MedSimEagle ${ }^{\circledR}$ ), simuladores virtuales, modelos animales, pacientes estandarizados o pacientes híbridos (combinación de un actor con un simulador de tareas). Para el entrenamiento de habilidades técnicas se dispone de salas para la práctica con piezas $e x$ vivo, modelos animales y diversos maniquíes comer- 
ciales de simulación (Laerdal ${ }^{\circledR}$ y Medical Simulator). Las actividades son impartidas por instructores especialmente formados en las teorías del aprendizaje experiencial y con un certificado, tras haber completado el curso de instructores en simulación clínica, expedido por el Institute for Medical Simulation (Cambridge, MA, Estados Unidos) [5]. También se dispone de técnicos para el manejo de los maniquíes y de actores con experiencia en actividades de simulación.

\section{Objetivos de la simulación}

- Facilitar el reconocimiento de los trastornos agudos y potencialmente graves más prevalentes de los sistemas cardiovascular y respiratorio.

- Facilitar el desarrollo del trabajo en equipo.

- Facilitar el aprendizaje del manejo de esos trastornos, incitando la toma de decisiones diagnósticas y terapéuticas en un entorno protegido y libre de riesgos.

- Facilitar la práctica reflexiva, considerando las propias fortalezas y carencias, tanto en conocimientos y habilidades como en aspectos emocionales.

- Facilitar la motivación de los estudiantes por el aprendizaje.

\section{Diseño de la actividad}

\section{Escenarios}

Se diseñaron ocho escenarios que recreaban pacientes con dolor torácico o disnea, bien en la planta de un hospital, bien en un servicio de urgencias. Los escenarios correspondían a cuatro diagnósticos (infarto de miocardio, embolia pulmonar, neumotórax, reagudización de broncopatía crónica), cada uno de los cuales se recreaba en dos escenarios con formas de presentación diferentes. Por ejemplo, un escenario recreaba un paciente con embolia pulmonar masiva central, y otro, un paciente con embolia periférica.

\section{Diseño}

Al diseñar los escenarios se definieron: el personal necesario (en general, un diplomado de enfermería actuando en el escenario, un técnico de manejo del maniquí y un profesor o instructor que guiaba el desarrollo del escenario y la reflexión posterior), el guión de la anamnesis (lo que el paciente decía), las variables de partida de la exploración (p. ej., tensión arterial, ruidos audibles a la auscultación), las pruebas complementarias disponibles y los cambios a efectuar en virtud de la actuación previsible (p. ej., aumentar la saturación de la hemoglobina si se administra oxígeno).

\section{Información previa}

Una semana antes de la fecha de realización de la simulación se citaba a todos los estudiantes a una sesión introductoria para explicar el desarrollo de la actividad y sus objetivos. Asimismo, se les planteaban los requisitos generales para su buen desarrollo (p. ej., confidencialidad sobre la actuación de los participantes y sobre las características de los casos simulados) y que la actividad no tenía un objetivo de evaluación del rendimiento de los estudiantes, sino favorecer el aprendizaje.

\section{Desarrollo del escenario}

Los estudiantes se dividieron en grupos de 6-8 sujetos, que a su vez se subdividieron en dos grupos de 3-4 estudiantes. Cada grupo de 6-8 estudiantes atendía dos escenarios con un diagnóstico determinado. Tras entrar en la sala, un subgrupo de 3-4 alumnos procedía a atender al paciente, mientras el otro subgrupo permanecía en la sala como meros observadores. Tras 15 minutos de actividad, se intercambiaban los papeles, de manera que el subgrupo observador pasaba a atender a un nuevo paciente, mientras que el otro subgrupo quedaba ahora como observador. Por tanto, los dos escenarios se desarrollaban a lo largo de 30 minutos en total.

\section{Reflexión}

Tras la fase de simulación, el grupo de 6-8 alumnos se reunía con un profesor durante 25-40 minutos para reflexionar sobre el caso. Los aspectos principales a tratar se habían definido previamente con el diseño de cada escenario e incluían cuestiones específicas para cada trastorno (p. ej., ¿cómo se pueden reconocer las complicaciones agudas de un infarto?) y otras cuestiones generales, tanto de tipo técnico (p. ej., reconocer la variabilidad de la presentación de una misma enfermedad) como emocional (p. ej., ansiedad experimentada, temores para la práctica futura, carencias percibidas) o relacional (p. ej., eficacia en la distribución de tareas). Al final se pasó una encuesta anónima con respuestas de tipo Likert y opción de comentarios libres (Tablas I y II).

\section{Resultados}

La actividad se desarrolló durante los cursos 2009 2010 y 2010-2011, habiendo participado 64 y 42 alumnos, respectivamente. En el primer periodo se consideró actividad obligatoria para todos los matriculados y los grupos eran de ocho alumnos, mientras que en el segundo periodo fue una actividad voluntaria y los grupos eran de seis alumnos. 
Tabla I. Encuesta 1.

1. La actividad me ha perecido útil para mi formación

2. Me ha parecido interesante

3. Previamente conocía algo sobre la simulación en medicina

4. Me he sentido en un ambiente confortable

5. Me ha resultado cansado

6. La organización de la actividad ha sido adecuada

7. Los objetivos de la actividad han sido claros

8. Dichos objetivos se han cumplido

9. Los escenarios me han parecido realistas

10. Es útil ver las propias actuaciones grabadas

11. Los análisis de los casos han sido bien llevados

12. Creo haber mejorado mis conocimientos sobre esa situación clínica

13. Creo que he mejorado mis habilidades y capacidad de aplicar los conocimientos en esa situación clínica

14. Recomendaría esta actividad a alumnos de otras universidades

Tabla II. Encuesta 2.

1. Cuando he tratado al paciente en el Hospital virtual Valdecilla he notado nerviosismo

2. El hecho de que me estén observando mientras cuido de los pacientes afecta al modo en que tomo decisiones

3. Me cuesta adaptarme a trabajar con un equipo de otros compañeros o enfermeros

4. No me resulta difícil cuestionar las decisiones de otros miembros del equipo con el que trabajo

5. Me preocupa no poseer los conocimientos necesarios cuando tenga que cuidar de un paciente

6. No se cómo voy a responder cuando me enfrento a un paciente grave

7. La limitación del tiempo disponible en una situación urgente dificulta mi capacidad para tomar decisiones clínicas

8. La experiencia de simulación me ha ayudado a conocer mejor cómo reacciono ante las situaciones de urgencia clínica

9. La experiencia de simulación me ha ayudado a entender el modo en que debo adquirir los conocimientos
Durante el curso 2009-2010, cada grupo actuó durante 30 minutos y la fase de reflexión duró $25 \mathrm{mi}$ nutos. Tras esa primera experiencia se consideró que el tiempo para la reflexión era insuficiente y se amplió a 40 minutos.

En la figura 1 se resumen los resultados de la encuesta llevada a cabo al finalizar la actividad. La valoración de los estudiantes fue muy positiva, tanto en cuanto al realismo de los escenarios como a la fase de reflexión, el interés general y la utilidad de la simulación. No hubo diferencias entre los dos periodos estudiados. Así, las puntuaciones promedio respecto a la 'utilidad' (pregunta 1) fueron de 4,8 y 4,9, respectivamente, en los dos años. Asimismo, en ambos periodos, la puntuación promedio para el 'interés' fue de 4,9 (pregunta 2).

Durante el segundo periodo se añadieron a la encuesta otras preguntas encaminadas a valorar algunos aspectos emocionales, como la ansiedad experimentada en el escenario simulado (Tabla II). Las respuestas se resumen en la figura $1 \mathrm{~b}$.

Para valorar la consistencia de los escenarios se compararon las respuestas a las encuestas de tres de ellos. Como se observa en la figura 2, no se encontraron diferencias relevantes en las respuestas a la encuesta 1 entre los estudiantes que habían participado en diferentes escenarios. Tampoco se encontraron diferencias en las respuestas a la segunda encuesta (datos no mostrados).

En los comentarios de texto libre de la encuesta, las sugerencias más comúnmente apuntadas fueron, por este orden, incrementar el número de actividades de este tipo a realizar durante el curso, comenzar a realizarlas en cursos anteriores de la licenciatura y aumentar el tiempo dedicado a cada simulación.

En cuanto a los recursos consumidos, cabe mencionar especialmente el tiempo de profesores e instructores. El tiempo dedicado al diseño de los escenarios clínicos fue de unas tres horas de un profesor y una hora de los otros profesionales. En cuanto a la simulación propiamente dicha, cada fracción de 1824 alumnos (en función de que los grupos fueran de seis u ocho estudiantes) precisó unas dos horas de trabajo del técnico que manejaba el simulador, dos horas de un enfermero que actuaba en el escenario y unas cuatro horas de dedicación de un profesor.

\section{Discusión}

Los resultados de esta experiencia piloto muestran que es factible utilizar la simulación clínica en la docencia pregraduada de la patología médica. Así mismo, los participantes refieren una elevada satis- 
facción con la actividad y creen que les resulta muy útil en su proceso de aprendizaje. Por tanto, es verosímil pensar que tiende a favorecer su motivación hacia el aprendizaje activo de conocimientos y su aplicación en el entorno clínico. Esa impresión positiva queda de manifiesto en la respuesta a la cuestión que refleja la impresión global (pregunta 14: 'recomendaría esta actividad a alumnos de otras universidades'), que obtuvo un promedio de 4,9 puntos sobre 5 .

En nuestros hospitales y centros de atención primaria, el papel de los estudiantes a menudo se limita al de simples observadores y resulta difícil que se impliquen activamente en la toma de decisiones diagnósticas y terapéuticas. Incluso cuando se incorporan de manera más activa a los equipos clínicos colaborando en la anamnesis o la exploración de los pacientes, no suelen tener responsabilidad directa sobre su manejo. Por ello, no es de extrañar que uno de los aspectos señalados como más positivos por los estudiantes en relación con la actividad de simulación ha sido la posibilidad de ser protagonistas, participando activamente en la toma de decisiones terapéuticas en un entorno 'casi real.'

La experiencia también ha resultado satisfactoria para los instructores. Ha permitido una observación directa de grupos pequeños de estudiantes actuando autónomamente, y así identificar lagunas en su formación y explorar aspectos emocionales y de trabajo en equipo que raramente se analizan en otros entornos. La importancia del análisis de estos factores durante la fase de reflexión (debriefing) viene remarcada por el hecho de que estos factores no técnicos, también denominados 'factores humanos', se han señalado como la causa principal de más del 70\% de las complicaciones de la atención sanitaria [6].

Nuestra experiencia nos lleva a pensar que la simulación clínica es factible en el pregrado y resulta satisfactoria. Desde el punto de vista de la eficacia, carecemos de datos propios, pero otros autores han demostrado la transferencia de las habilidades adquiridas en simulación al entorno clínico real, aumentado el grado de retención de lo aprendido cuando se compara con los métodos docentes tradicionales [7-9]. Los estudiantes de medicina expuestos a una sesión de simulación clínica obtuvieron mejores resultados en los exámenes que los controles, y la diferencia persistía un año después [10].

La simulación puede ser un buen complemento a las prácticas en un entorno clínico real, no un sustituto de ellas, pues la experiencia de la realidad posee matices que no son reproducibles en un entorno simulado. Pensamos que la simulación puede integrarse dentro del currículo de las facultades de medicina
Figura 1. Respuestas a la encuesta 1 en dos años sucesivos (a) y a la encuesta 2 durante el segundo año (b). Las barras indican el promedio de las respuestas, en una escala de 1 a 5 , en función del grado de acuerdo con el enunciado.

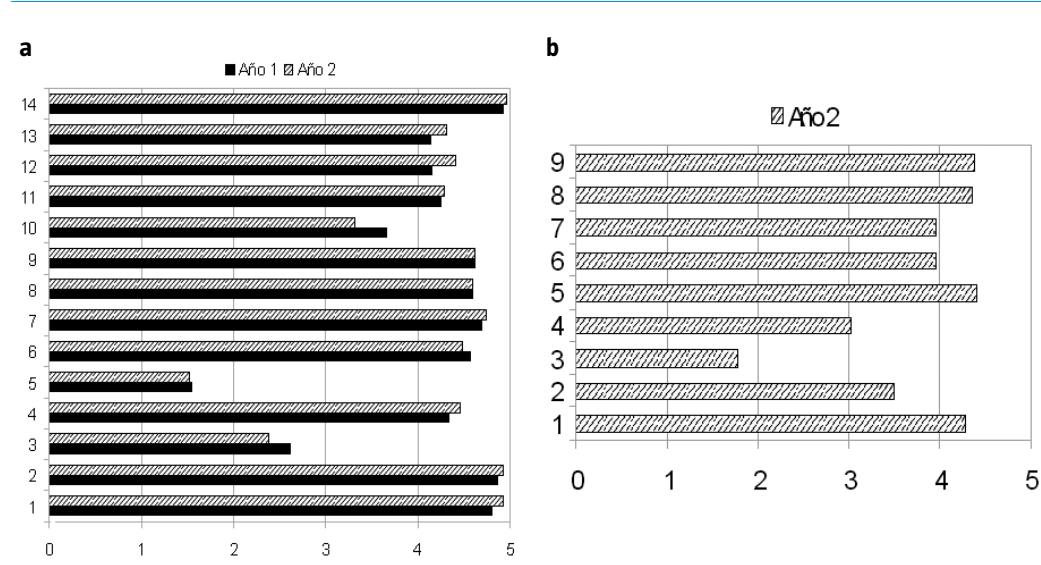

Figura 2. Respuestas a la encuesta 1 por grupos de alumnos expuestos a tres escenarios diferentes. Las barras indican el promedio de las respuestas, en una escala de 1 a 5 , en función del grado de acuerdo con el enunciado.

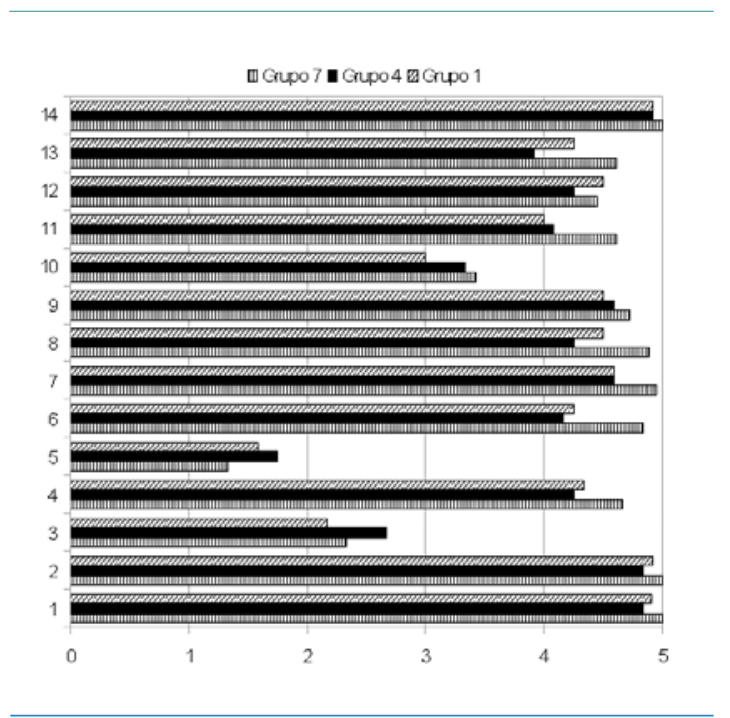

de manera coste-efectiva, 'dando vida' de forma sistemática y programada a las situaciones clínicas más relevantes de cada asignatura. Desde nuestra perspectiva, algunos de los objetivos de las actividades de simulación en el pregrado pueden ser:

- Garantizar que los estudiantes son capaces de identificar y manejar situaciones graves y relativamente comunes que requieren atención inmediata. 
- Facilitar la retención de conocimientos y habilidades a través de su aplicación práctica.

- Experimentar, reflexionar y, en consecuencia, manejar mejor el estrés para optimizar la toma de decisiones en situaciones graves.

- Desarrollar habilidad para el trabajo cooperativo en situaciones de crisis.

- Identificar lagunas en el conocimiento o habilidades, que deben abordarse prioritariamente para mejorar el propio aprendizaje.

Para conseguir esos objetivos parece necesario, como apuntaban los propios estudiantes, repetir en varias ocasiones la actividad. Los alumnos a los que se les ofrece esta oportunidad de implicarse en la práctica deliberada y repetitiva con simulación parecen adquirir las habilidades necesarias en periodos más breves que aquellos que se exponen únicamente a las situaciones clínicas rutinarias con pacientes reales. De hecho, existe relación entre el tiempo total de exposición a actividades de simulación y los resultados de aprendizaje $[11,12]$. Que los estudiantes participen en escenarios simulados de alto realismo como los descritos en este trabajo, en unas cuatro o cinco ocasiones a lo largo del curso, parece un compromiso razonable entre lo ideal y lo factible. A la vista de los resultados, no parece haber diferencias importantes entre los grupos de seis y de ocho alumnos, ni tampoco parece que los índices de satisfacción con la actividad varíen en función de que sea una actividad obligatoria u optativa.

Con independencia de la tecnología y sofisticación de los materiales utilizados en la simulación, la fase de reflexión estructurada tras cada escenario desempeña un papel fundamental. La metodología docente que utiliza la simulación clínica se basa en las teorías de Kolb sobre el aprendizaje del adulto. Se propone vivir una experiencia concreta e inmediata en la que el participante pone en práctica sus habilidades y conocimientos para resolver diferentes problemas clínicos. Posteriormente, los participantes reflexionan de manera activa sobre la toma de decisiones y la resolución del problema vivido; se integran las reflexiones de la fase anterior y se forma un modelo teórico. Más tarde se ponen a prueba los conceptos incorporados en situaciones nuevas. Este ciclo tiene lugar tanto con los casos simulados como con los vividos en el entorno clínico real [13].

Por tanto, el instructor desempeña un papel esencial durante el diseño de los escenarios y durante la fase de reflexión, pues facilita y modera el pensamiento analítico de los participantes. Debe ser capaz de alcanzar los objetivos definidos a priori a través del análisis del desarrollo del escenario con- creto. Debe aportar información de retorno a los estudiantes sobre sus logros y sus deficiencias, en un entorno abierto, respetuoso y participativo. Para ello es útil recordar otras características del aprendizaje de los adultos [5]:

- Necesitan saber para qué están aprendiendo.

- Se motivan cuando se plantea resolver un problema.

- Construyen su aprendizaje sobre la base de las experiencias previas, que deben respetarse.

- El enfoque docente debe adaptarse a sus conocimientos previos.

- Deben participar activamente en el proceso de aprendizaje.

Asimismo, deben mostrarse no sólo las deficiencias, sino las cosas bien hechas, reforzando la confianza y facilitando que puedan repetirse de modo consciente en una situación similar posterior [14]. Para ello, a menudo se ha utilizado una aproximación sugerida por Pendleton, con las fases siguientes:

- El instructor pide a los estudiantes que identifiquen qué han hecho bien.

- El instructor expone lo que cree que han hecho bien.

- El instructor pide a los estudiantes que identifiquen qué aspectos deberían mejorar.

- El instructor indica qué aspectos deberían mejorar.

- Estudiantes e instructor elaboran un plan de mejora de las deficiencias.

En definitiva, el análisis permite elaborar nuevos modelos mentales y redirigir nuestras acciones durante la práctica profesional con el fin de mejorar los resultados clínicos [15].

Frente a este potencial de aplicación de la simulación clínica en el pregrado, su implementación no está exenta de retos. La simulación no es una tecnología, sino una técnica o método de aprendizaje centrado en el participante y basado en la propia experiencia, y en nuestra opinión el elemento clave es la formación de los instructores. Así, la principal limitación para su aplicación generalizada son los costes derivados de su formación en la metodología docente, y el tiempo dedicado por ellos y los participantes a cada actividad en particular. De acuerdo con el diseño de nuestra actividad, se precisa una jornada completa de un profesor, un técnico y un enfermero para que un colectivo de unos 40 estudiantes se exponga a dos casos clínicos. Se requieren además unas tres horas de un profesor y una hora de los profesionales de simulación para el diseño previo de los casos, la recopilación de los materiales y el ensayo de los escenarios. Además, la fi- 
nanciación ha de considerar los costes directos de adquisición y mantenimiento de los sistemas de simulación y los espacios físicos. La integración de esta nueva metodología implica también un cambio en la cultura de la formación de pregrado y definir los criterios que permitan seleccionar las competencias que mejor se beneficien de este tipo de entrenamiento.

Finalmente, es necesario advertir del riesgo de resultar 'deslumbrados' por la sofisticación de algunas tecnologías, de manera que impidan reconocer la importancia de otros factores. Como han señalado previamente otros grupos, los resultados de la educación basada en la simulación dependen del producto de tres factores: medios técnicos adecuados, profesores entusiastas preparados para sacarles el máximo partido e integración en el currículo [16]. Sólo la combinación inteligente, adaptada a las circunstancias propias de cada institución, de actividades de simulación de alto realismo con las prácticas focalizadas en aspectos más concretos (p. ej., los simuladores de tareas para el aprendizaje de habilidades técnicas simples) y la experiencia clínica real contribuirá a obtener resultados de aprendizaje óptimos.

\section{Bibliografía}

1. Ziv A. Simulators and simulation-based medical education. In Dent JA, Harden RM, eds. A practical guide for medical teachers. Edinburgh: Churchill-Livingstone; 2009. p. 217-22.

2. Palés-Argullós JL, Gomar-Sancho C. El uso de las simulaciones en educación médica. Teoría de la educación. Educación y Cultura de la Sociedad de Información 2010; 11: 147-69.

3. Sancho R, Rabago JL, Maestre JM, Del Moral I, Carceller JM. Integración de la simulación clínica en el programa formativo de la especialidad de Anestesiología y Reanimación. Rev Esp Anestesiol Reanim 2010; 57: 656-63.

4. Del Moral I, Rabanal JM, Díaz de Terán C. Simuladores en anestesia. Rev Esp Anestesiol Reanim 2011; 48: 415-22.

5. Okuda Y, Bryson EO, DeMaria S Jr, Jacobson L, Quinones J, Shen B, et al. The utility of simulation in medical education: what is the evidence? Mt Sinai J Med 2009; 76: 330-43.

6. Kohn LT, Corrigan JM, Donaldson MS. To err is human. Building a safer health system. Washington DC: National Academic Press; 2000.

7. Kuduvalli PM, Parker CJ, Leuwer M, Guha A. Retention and transferability of team resource management skills in anaesthetic emergencies: the long-term impact of a high-fidelity simulationbased course. Eur J Anaesthesiol 2009; 26: 17-22.

8. Fraser K, Wright B, Girard L, Tworek J, Paget M, Welikovich L, et al. Simulation training improves diagnostic performance on a real patient with similar clinical findings. Chest 2011; 139: 376-81.

9. Sturm LP, Windsor JA, Cosman PH, Cregan P, Hewett PJ, Maddern GJ. A systematic review of skills transfer after surgical simulation training. Ann Surg 2008; 248: 166-79.

10. Gordon JA, Brown DF, Armstrong EG. Can a simulated critical care encounter accelerate basic science learning among preclinical medical students? A pilot study. Simul Healthc 2006; 1 (special number): 13-7.

11. Issenberg SB, Scalese RJ. Best evidence on high-fidelity simulation: what clinical teachers need to know. Clin Teach 2007; 4: 73-7.

12. Lammers RL, Davenport M, Korley F, Griswold-Theodorson S, Fitch MT, Narang AT, et al. Teaching and assessing procedural skills using simulation: metrics and methodology. Acad Emerg Med 2008; 15: 1079-87.

13. Davidson M. The taxonomy of learning. Int Anesthesiol Clin 2008; 46: 1-15.

14. Gordon J. ABC of learning and teaching in medicine: one to one teaching and feedback. BMJ 2003; 326: 543-5.

15. Rudolph JW, Simon R, Dufresne RL, Raemer DB. There's no such thing as 'nonjudgmental' debriefing: a theory and method for debriefing with good judgment. Simul Healthc 2006; 1: 49-55.

16. McGaghie WC, Siddall VJ, Mazmanian PE, Myers J. Lessons for continuing medical education from simulation research in undergraduate and graduate medical education: effectiveness of continuing medical education: American College of Chest Physicians Evidence-Based Educational Guidelines. Chest 2009; 135: S62-8. 